Dr Sudish Murthy (Cleveland, Ohio). The audience has to recognize that induction chemotherapy is a different breed of cat than induction chemoradiation therapy, and that is where most of our data are generated from. The other thing about propensity analysis is that it takes a fair bit of bias of thinking what is going to affect the outcome in this patient group to come up with the variables that you need to impute into these algorithms. The fewer the variables, the less strenuous your matching algorithms are. When you have these large databases, they are only matching on 5 variables?

Dr Thomas. Nine variables.

Dr Murthy. With only 9 variables, you will no doubt lose some granularity in terms of pseudo-randomization.

\title{
EDITORIAL COMMENTARY
}

\section{A French connection}

\author{
Sudish C. Murthy, MD, PhD
}

See related article on pages 73-83.

Perhaps it's time for the STS (Society of Thoracic Surgeons) and SEER (Surveillance, Epidemiology, and End Results) databases to move over: There's a new sheriff in town. This issue contains a large study of pneumonectomy patients from France, the substrate of which is the EPITHOR database (French Society of Thoracic and Cardiovascular Surgery database). Data on nearly 4500 patients were able to be accessed, and we are all witnesses to the fruits of mining this database. The French report a nationwide mortality of just below $8 \%$ for pneumonectomy and identify the risks of early mortality and a few curious protective factors. The sheer size of the study immediately makes it interesting, regardless of the actual findings.

Some bias seems to exist against large database studies generated outside the United States, especially when the findings reflect a better outcome for patients than we report from our own US national registries or databases. Lung cancer survival in Japan trumps that in the United States on a stage-for-stage basis. The same could be said for management of gastric cancer. Perhaps we in the United States find this insulting and worry that it somehow brings our own capabilities into question. Our usual response to these types of reports is to write editorials that point out some ambiguity in the database, or mistake in the statistical analysis, as a principal reason for the survival advantage. Unfortunately, we often ignore the expert care and

\footnotetext{
From the Cleveland Clinic Foundation, Cleveland, Ohio.

Disclosures: Author has nothing to disclose with regard to commercial support.

Received for publication Oct 22, 2014; accepted for publication Oct 22, 2014

Address for reprints: Sudish C. Murthy, MD, PhD, Cleveland Clinic Foundation/Desk

J4-1, Thoracic and Cardiovascular Surgery, 9500 Euclid Ave, Cleveland, OH

44195 (E-mail: murthys1@ccf.org).

J Thorac Cardiovasc Surg 2015;149:83-4

$0022-5223 / \$ 36.00$

Copyright (c) 2015 by The American Association for Thoracic Surgery

http://dx.doi.org/10.1016/j.jtcvs.2014.10.098
}

management of these patients and fail to consider that the presentation of the disease and response to therapy might simply be different elsewhere in the world.

To evaluate this possibility, the French database EPITHOR has served as the basis for a study of pneumonectomy for lung cancer. This data repository, as well as those created elsewhere in Europe, is part of a greater quality initiative and represents an attempt to standardize care across the country. EPITHOR is more than a registry, and surprisingly, contains fairly detailed information on postoperative complications that is used in this study. Because of the relative granularity of the database, the authors were able to perform modern comparative analytic techniques, including propensity matching, to fortify their conclusions. Most of their findings are in keeping with common sense and are not grossly dissimilar from results reported in this country, which I'm sure will be a relief to our US readership. A nationwide mortality of slightly less than $8 \%$ (over the past decade) is commendable. Risks for poor early outcome include age, low body mass index (BMI, a suspected surrogate for malnutrition), and poor performance status.

Curiously, these French authors also identify induction therapy and "overweight" BMI as "protective factors" after pneumonectomy. I suspect this should not be taken literally by the reader as an injunction to fatten up one's patients and inject them with systemic poisons just before taking them out to the proverbial woodshed for an old-fashioned beating to optimize outcome. Rather, these variables are likely surrogates for a more robust and hardy stock, and as with all large databases, this is where the facts end, and the speculation, conjecture, and innuendo begin.

It is easy to lose one's clinical perspective during a large number-crunching exercise such as this. "Statistics this" and " $P$ value that" are great up to a certain point, at which clinical gestalt must reign supreme. French "overweight" is probably the equivalent of American "healthy." Most in the 
United States would consider themselves fortunate to operate on a patient in this weight category (BMI, $25-30 \mathrm{~kg} / \mathrm{m}^{2}$ ). Of course, the French, with their high-fashion mentality, might take umbrage at this kind of "figure," but that description would seem to reflect a patient who is likely to be in a reasonable nutritional state and without muscle wasting, and I think that is precisely the surrogate that this variable represents.

The protective effect of induction therapy is a bit harder to rationalize and understand. How could pretreating (and debilitating) a patient before pneumonectomy ever be a good thing in regard to improved early postoperative outcomes? That concept certainly seems inconceivable. Moreover, randomized data have demonstrated that induction therapy has the exact opposite effect on cancer pneumonectomy outcome. ${ }^{1}$ I suspect an explanation resides in what is not in the EPITHOR database.

Induction therapy for lung cancer in France is not the same animal as it is in the United States. Randomized studies in the United States generally compare outcomes of patients treated with induction chemoradiotherapy and not simply induction chemotherapy. The addition of the radiation therapy is probably what gives the patient the real punch in the stomach. The authors fully disclose this fact in their discussion. Even so, induction chemotherapy is no doubt still a difficult hurdle for these pneumonectomy patients to surmount.

For patients with stage IIIa non-small cell lung cancer to be offered induction therapy, they must have passed, certainly in my practice, the dreaded "eyeball" test. If they do not pass, they are generally offered definitive chemoradiotherapy and no resection, because any possible benefit of resection in this setting is realized only if patients recover from their entire treatment regimen. Consequently, when we look back on who is actually offered aggressive multimodality therapy for locally advanced lung cancer, we are always surprised at what a robust cohort we have selected.

What makes for a good eyeball test is hard to say, but I suspect it's some amalgam of excellent nutritional state, robust musculature, excellent gas exchange, sufficient pulmonary parenchyma to sacrifice, tobacco cessation, and a positive mental outlook, among other things. What might be captured in a database that reflects these factors? Perhaps grip strength, walk oximetry, oxygen consumption during exercise, serum prealbumin and transferrin levels, quantitative perfusion scans, normal preoperative pulmonary artery pressures, and a multidisciplinary consortium of physicians and surgeons independently arriving at the same conclusion. Thus, my impression is that induction therapy (chemotherapy) has selected a much more robust cohort of French people who are able to battle through their recovery despite being burdened by the preoperative chemotherapy.

We now add EPITHOR to the growing list of large databases. There is little doubt that the quality of lung cancer care will improve, especially if the lessons learned from its analysis are used to change practice patterns in France. Data miners need to be aware of the limitations of any large database, and not necessarily let the statistics alone tell the whole story. As we learn what some of these broad variables actually represent, future iterations of data repositories should become increasingly more informative. If we fail to ever figure out this answer key, then maybe we shouldn't interpret findings so literally.

\section{Reference}

1. Albain KS, Swann RS, Rusch VW, Turrisi AT III, Shepherd FA, Smith C, et al. Radiotherapy plus chemotherapy with or without surgical resection for stage III non-small-cell lung cancer: a phase III randomised controlled trial. Lancet. 2009;374:379-86. 